\title{
Optimum Dataset Size and Search Space for Minimum Zone Roundness Evaluation by Genetic Algorithm
}

\author{
A. Meo, L. Profumo, A. Rossi, M. Lanzetta \\ Department of Civil and Industrial Engineering, \\ University of Pisa, Largo Lazzarino, 56122 Pisa, Italy, lanzetta@unipi.it
}

\begin{abstract}
Roundness is one of the most common features in machining. The minimum zone tolerance (MZT) approach provides the minimum roundness error, i.e. the minimum distance between the two concentric reference circles containing the acquired profile; more accurate form error estimation results in less false part rejections. MZT is still an open problem and is approached here by a Genetic Algorithm. Only few authors have addressed the definition of the search space center and size and its relationship with the dataset size, which greatly influence the inspection time for the profile measurement and the convergence speed of the roundness estimation algorithm for a given target accuracy. Experimental tests on certified roundness profiles, using the profile centroid as the search space center, have shown that the search space size is related to the number of dataset points and an optimum exists, which provides a computation time reduction up to an order of magnitude.
\end{abstract}

Keywords: Minimum zone tolerance (MZT), roundness error, genetic algorithm, CMM

\section{INTRODUCTION}

$\mathrm{T}$ HE GROWING demand for efficiency and productivity, the increasing complexity of manufactured parts and assembly tasks require high-speed inspection.

Form errors play a major role in quality control and product conformance to the designed tolerances.

Common one and three dimensional form tolerances are respectively straightness and cylindricity, sphericity, and flatness. Roundness (also known as circularity) is a typical two dimensional feature to be inspected. Form tolerance is evaluated from a set of measured points on the product profile, with reference to an ideal geometric feature, i.e. a circle in the examined case. The most used criteria to establish the reference circle are: the Least Squares method (LSQ), the Maximum Inscribed Circle (MIC), the Minimum Circumscribed Circle (MCC) and the Minimum Zone Tolerance (MZT). The use of a particular data fitting method depends on the application, e.g. MIC (peg) and MCC (hole) can be used when mating (peg-in-hole) is involved. LSQ is one of the methods used by Coordinate Measuring Machines (CMM). It is efficient in computation and is fast also with a high number of measured points, but the roundness error is higher than that determined by the other mentioned methods. Therefore, potentially good parts can be rejected resulting in an economic loss. In this regard, as expressed by its name, the minimum zone (MZ) approach can be considered the best estimation of the roundness error and it meets the definition of roundness error according to international standards [1] [2].

The MZT determines two concentric circles that contain the measured profile and such that the difference in radii is the least possible value. As shown in Fig.1, $C_{1}\left(x_{1}, y_{1}\right)$ and $C_{2}\left(x_{2}, y_{2}\right)$ are two possible centers of two concentric circles that include the measured points and $\Delta r_{1}\left(x_{1}, y_{1}\right)<\Delta r_{2}\left(x_{2}, y_{2}\right)$ are their difference in radii. By finding the features in Fig.1, the MZ error and the related MZ center are determined. However, the MZT is a non linear problem and several methods to solve this problem have been proposed in the literature: (i) computational geometry techniques and (ii) the solution of a non linear optimization problem. The first approach is, in general, very computationally expensive, especially, when the number of data points is high. One of these methods is based on the Voronoi diagram [3].

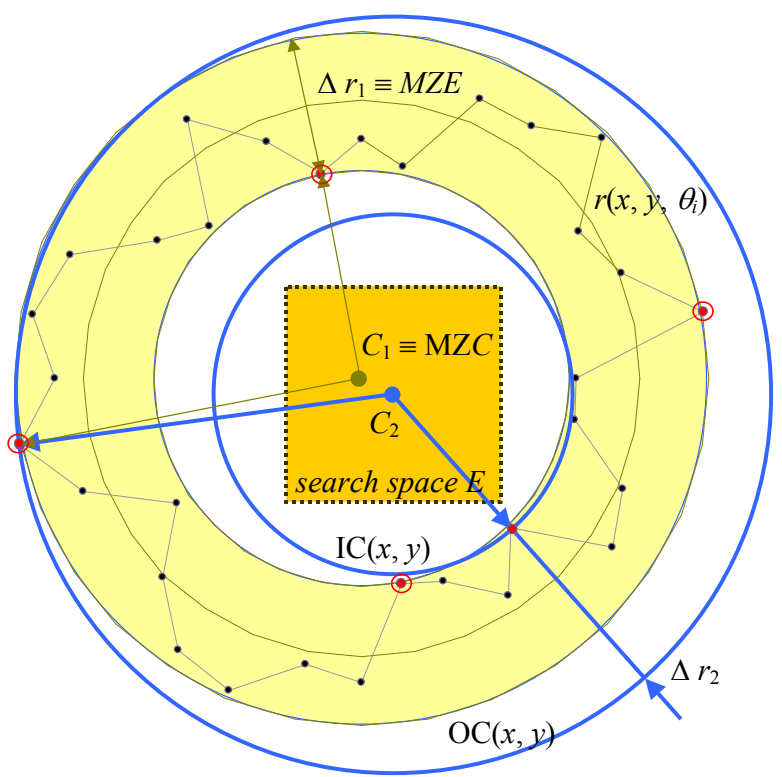

Fig.1. MZ error. $C_{1}$ and $C_{2}$ are possible locations of the centers of the two concentric circles. $\Delta r_{1}$ and $\Delta r_{2}$ are the difference in radii. If the minimal difference in radii is $\Delta r_{1}$, that is the MZ error and $C_{1}$ is the MZ center. The dotted square search space $S_{r\left(x, y, \theta_{i}\right)}$ with side $2 E$ is centered on the centroid of the acquired profile.

Considering the trend towards higher number of acquired data points, in the order of thousands, made possible by optical methods or CMM scanning, this work addresses the latter approach: non-linear optimization. Among 
optimization techniques are Chebyshev approximation [4] and simplex search [5]. Metaheuristics approaches are also available in the literature, such as particle swarm optimization (PSO) [6], linear approximation [7], and genetic algorithms (GAs) [3], [8], [9], [10] and [11]. Performance of methods has been reviewed in [12].

Genetic algorithms are widely used in research for nonlinear problems. They are powerful and can be easily implemented being a general-purpose optimization tool. Possible solutions are processed concurrently (in the order of hundreds) and converge to a local optimum, which is very close or coincident to the problem optimum solution, by user selectable rules, such as elitist and random selection.

This paper explores two major aspects of the inspection strategy: selecting the number of acquired datapoints and the search space size for the minimum zone center, whose coordinates represent the genes of the GA.

In industrial inspection, the measurement accuracy is fixed, because it depends on the manufacturing process (e.g. CNC machining, forming) and on the measuring instrument (e.g. CMM, dial gage, optical scanner, vision system). The accuracy range explored in this paper is that commonly available in traditional manufacturing, between 0.01 and 0.2 $\mathrm{mm}$.

It will be shown experimentally that a lower minimum zone error $M Z E$ (higher accuracy) is achieved by a higher number of data points. However this causes an increase of acquisition time and cost and consequently the optimal number of data points needs to be properly selected.

The influence of sampled data point number and search space size on processing time and accuracy will also be discussed, and a method to select their optimal values for a given target accuracy in the estimation of the MZ center will be provided.

One inconvenience of the minimum zone approach is the presence of local minima, for this reason the search space, which should be defined when using genetic algorithms, should be carefully selected in order to reduce the computation time.

\section{STATE OF THE ART ON SAMPLING DATA POINTS AND SEARCH SPACE SIZE}

Only few contributions are available in the literature regarding the optimal selection of the number of sample points and the search space size and position, particularly with genetic algorithms.

Moroni and Petro [12] propose a technique to speed up the exhaustive generation of solutions (brute force algorithm) which starts with a single point and increases one sample point at each step in order to generate all the possible subsets of points, until the tolerance zone of a subset covers the whole dataset (essential subset).

In [13], the increase of data points shows to be effective only up to a given limit. Recommended dataset sizes are given for different data fitting methods (LSQ, MIC, MCC, MZT) and for three different out-of-roundness types (oval, 3-lobing and 4-lobing). In particular, for the MZT the recommended numbers are 48, 42, 38 points, for the three out-of-roundness types respectively. However, no indications are provided about the adopted algorithms. Similar approaches are in [14] and [15].

The strategy to equally spaced points sampled on the roundness profile is generally adopted in the literature. Conversely, in previous works, the authors developed a cross-validation method for small samples to assess the kind of manufacturing signature on the roundness profile in order to detect critical points such as peaks and valleys [16] [17]. They use a pre-sampling strategy to locate peaks and valleys where the sampling density is increased. Weckenmann et al. simulated the impact of the number and distribution of sampled points (sampling strategy) for the clamping strategy, functional assessment and operator influence [18] and their use by the data fitting algorithm [19].

In a genetic algorithm for roundness evaluation, the center candidates are usually the individuals of the population (chromosomes). The search space is an area enclosed by the roundness profile where the center candidates of the initial population are selected for the data-fitting algorithm. The area is rectangular because the crossover operator changes the $x_{i}$ and $y_{i}$ coordinates of the parents to generate offspring [3]. After crossover, according to Lemma 1 in [11] the $x_{i}$ and $y_{i}$ coordinates of next generation are located at the rectangle circumscribed to $S_{r\left(x, y, \theta_{i}\right)}$.

In order to find the $\mathrm{MZ}$ error, the search space must include the global optimum solution i.e. the MZ center. The center of the rectangular area is an estimation of the MZ center evaluated as the mean value of the $x_{i}$ and $y_{i}$ coordinates of the sampled points [3], [8], [9], [10] and [11]. In [8] the search space is a square of fixed $0.2 \mathrm{~mm}$ side, in [11] it is $5 \%$ of the circle diameter. In [3], it is determined by the distance of the farthest point and the nearest point from the centroid. In [9] it is the rectangle circumscribed to the sample points. The authors [20] proposed a search space size given by the theoretical upper bound for the centroid to minimum zone center distance. This upper bound is based on a worst-case geometrical feature formed by two concentric-opposite arcs. The search space size is $\pi^{-1} E_{C}$, where $E_{C}$ is the roundness error related to the centroid (the search space center) and it can be evaluated in closed form.

From this analysis, further investigation is required for the optimum dataset size and search space for minimum zone roundness evaluation by a genetic algorithm. In addition, to the best of our knowledge, no previous works are available about testing genetic algorithms for the roundness error evaluation with combined tests between different dataset and different search space sizes, particularly with large datasets.

\section{Problem Formulation}

The MZ error is the solution of the following optimization problem [11]:

$\min \left[\max _{\theta_{i}=i \times \frac{2 \pi}{n}, i=1, . ., n} r\left(x, y, \theta_{i}\right)-\min _{\theta_{i}=i \times \frac{2 \pi}{n}, i=1, . ., n} r\left(x, y, \theta_{i}\right)\right]$

subject to $(x, y) \in S_{r\left(x, y, \theta_{i}\right)}$

where $\theta_{i}=i \times \frac{2 \pi}{n}, i=1, \ldots, n$ are a number of equally-spaced points of the roundness profile $r\left(x, y, \theta_{i}\right)$ of the reference 
circle of center $(x, y)$ and radius $R ; S_{r\left(x, y, \theta_{i}\right)}$ is the search space, which is function of the sampled points $\theta_{i}$ and their dataset size $n$.

The center of the search space is computed as an average value of the measuring points coordinates from the literature (centroid of the roundness profile):

$$
\vec{C}_{n}=\left(\frac{1}{n} \sum_{i=1}^{n} x_{i}, \frac{1}{n} \sum_{i=1}^{n} y_{i}\right)
$$

Also

$$
M Z E=\min _{(x, y) \in E} \mathrm{OC}(x, y)-\mathrm{IC}(x, y)
$$

where: $(x, y)$ are the coordinates of the center of the two concentric circles included in the square of side $E$, also termed the search space (where the possible solution may be within); $\mathrm{OC}(x, y)$ and $\mathrm{IC}(x, y)$ are respectively the maximum and the minimum distance of the center $(x, y)$ from the data points. So, the algorithm has to find the optimum center $(x, y)$ in order to minimize that loss function leading to the $M Z E$, i.e. a sufficiently accurate estimate of the roundness error. For every point of the circular profile acquired by a CMM, the distance from the center $(x, y)$ is computed.

\section{THE PROPOSED GENETIC ALGORITHM APPLIED TO THE MZT PROBLEM}

Genetic algorithms constitute a class of search methods especially suited for solving complex optimization problems. Genetic algorithms maintain a population of individuals, which represent possible solutions of the given problem. The individuals are represented by their chromosomes, which are made of genes, the inheritable properties of individuals. Genetic algorithms operate on genes by means of genetic operators. At each generation, genetic operators are applied to selected individuals of current population in order to create a new generation of individuals. The selection of individuals is obtained according to a fitness function, which reflects how well a solution fulfills the requirements of the given problem, e.g. the loss function to be optimized.

The genetic algorithm used to solve the MZT problem has been designed as in [11] where it has been shown that a careful selection of the genetic parameters, may lead to a reduction of the processing time by up to one order of magnitude allowing real-time operation. By analyzing the available literature, the proposed genetic parameters, also reported in this paper, are valid for the roundness problem over a wide range of numbers of data points. The parameter meaning, use and value is listed in Table 1.

This algorithm has a starting population of 70 elements $\left(P_{s}\right)$, randomly chosen in a square search space, centered in the centroid, with variable side $2 E$. The population elements are the possible centers of the MZ circles. Therefore 70 possible centers with their $\mathrm{OC}(x, y), \mathrm{IC}(x, y)$ and $\mathrm{MZ}$ error are simultaneously evaluated at each iteration of the algorithm. The evaluation of expression (3) for every center corresponds to its fitness value, chosen to estimate the circular profile roundness.

\section{COMPUTATION EXPERIMENTS}

Datasets used in computation experiments are generated with NPL Chebyshev best-fit circle certified software [21]. The use of artificially generated datasets versus datasets obtained from real parts has the following benefits:

- the circle center is known;

- errors are randomly distributed, so results are more general, e.g. not manufacturing signature specific.

Datasets generated have maximum residual deviation from 0.01 to $0.09 \mathrm{~mm}$, and sizes up to 10,000 points. The maximum residual deviation is equal to half of the exact $\mathrm{MZ}$

\begin{tabular}{|c|c|c|c|c|}
\hline Parameters & Type & Symbol & Value & Description \\
\hline dataset size & G & $n$ & $10-10,000$ & number of sample points on the circumference \\
\hline search space & G & $E$ & $0.1-10$ & initial population randomly selected within a square of side $2 E[\mathrm{~mm}]$ \\
\hline $\begin{array}{l}\text { population } \\
\text { size }\end{array}$ & A & $P_{s}$ & 70 & set of chromosomes used in evolving epoch \\
\hline selection & A & & $\begin{array}{l}\text { elitist } \\
\text { selection }\end{array}$ & $\begin{array}{l}\text { individuals sorted based on their fitness function. The best individuals produce offspring. The } \\
\text { next generation will be composed of the best chromosomes chosen between the set of } \\
\text { offspring and the previous population }\end{array}$ \\
\hline crossover & A & $P_{c}$ & 0.7 & $\begin{array}{l}\text { one point crossover: new individuals created as component-wise linear combination from two } \\
\text { among the } p c \times p o p \text { parents' genes (i.e. coordinates) with high fitness at each generation }\end{array}$ \\
\hline mutation & A & $P_{m}$ & 0.07 & $\begin{array}{l}\text { mutation is a source of variability, prevents the search to be trapped in local minima and is } \\
\text { applied in addition to selection and crossover. A new individual is created by making } \\
\text { modifications to one selected individual, based on the mutation probability. pm } \times \text { pop } \\
\text { individuals are modified by changing one gene (i.e. coordinate) with a random value }\end{array}$ \\
\hline chromosome & A & $x, y$ & float & center coordinates \\
\hline stop criterion & A & $N_{\text {stop }}$ & 100 & $\begin{array}{l}\text { the algorithm has an iterative behavior and needs a stop condition to end the computation. } \\
\text { Possible criteria include: overcoming a predefined threshold for the fitness function or } \\
\text { iteration number or their combinations. The algorithm computes } N_{\text {stop }} \text { iterations (generations) } \\
\text { after the last best roundness error evaluated rounded off to the fourth decimal digit }(0.1 \mu \mathrm{m})\end{array}$ \\
\hline
\end{tabular}

Table 1. Algorithm parameters and type (G for geometric, A for algorithmic) from [10]. 


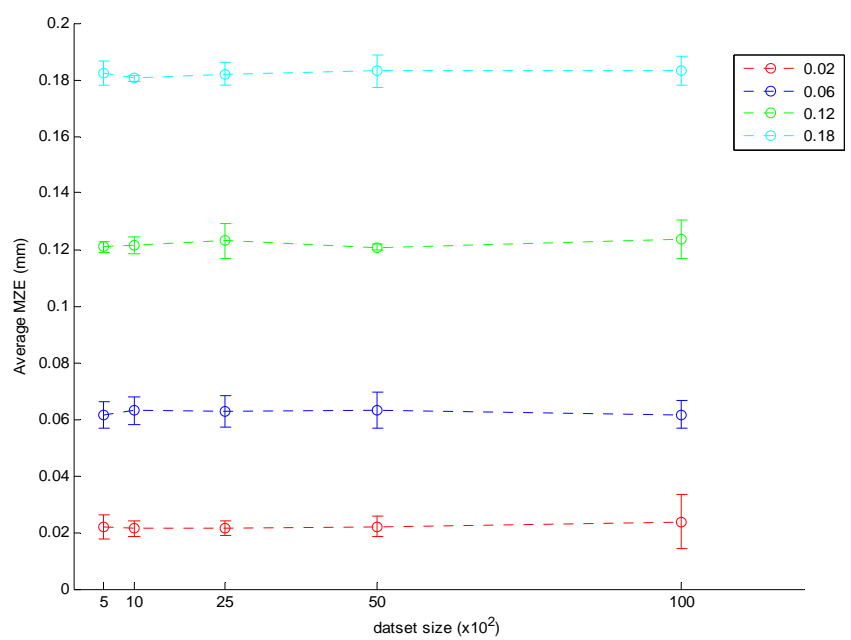

Fig.2. Average $\mathrm{MZ}$ error with error bars from 30 repetitions versus dataset size $(500<$ datapoints $<10,000)$ for different roundness errors. Individual trends are available in Appendix.

error. Without loss of generality, datasets have fixed center in the axis's origin $(0,0)$ and radius $R$ equal to $20 \mathrm{~mm}$. The algorithm is executed 30 times for each test, and the average MZ error, with its standard deviation and the average computation time with $1.2 \mathrm{GHz}$ Pentium M based PC are evaluated.

In the first set of experiments the described genetic algorithm is tested with large datasets (between 500 and 10,000 acquired datapoints) in order to evaluate how the $\mathrm{MZ}$ error changes and whether any improvements occur.

The average and standard deviation of the minimum zone error found with the genetic algorithm versus the number of points increase for datasets with different roundness errors are displayed in Fig.2, (and detailed from Fig.10 to Fig.13 in Appendix). Similarly, the computation time is displayed versus the dataset size in Fig.3.

No significant improvement occurs, when the dataset size is large, whilst the computation time has a linear rising trend.

In the next set of computation experiments, the upper



Fig.3. Average computation time from 30 repetitions versus dataset size $(500<$ datapoints $<10,000)$ for different roundness errors.

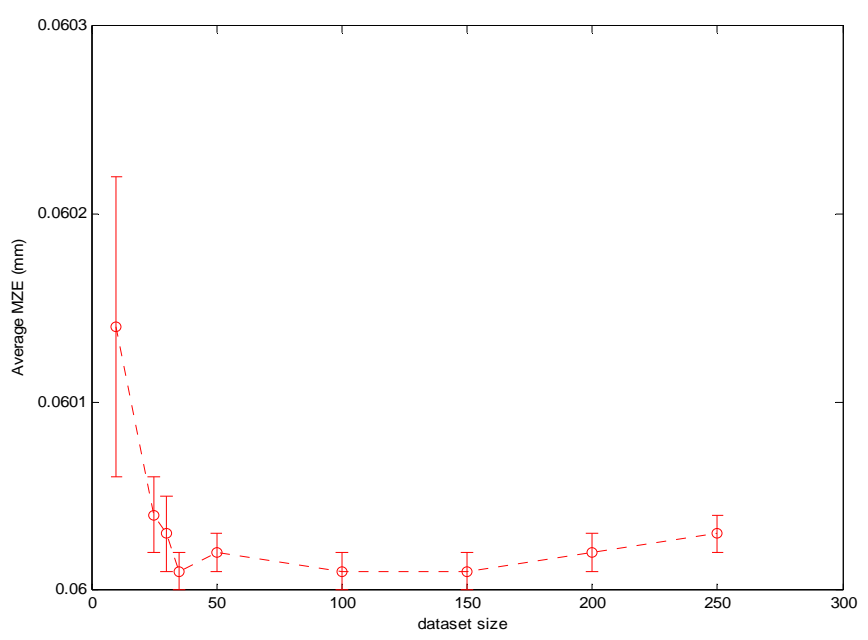

Fig.4. Average MZ error with error bars from 30 repetitions versus dataset size (datapoints $<500$ ) for roundness error of $0.06 \mathrm{~mm}$.

limit for the dataset size is kept at 500 points, because no benefit is found beyond that, and the roundness error is fixed at $0.06 \mathrm{~mm}$.

From Fig.4, the average minimum zone error shows a minimum with dataset size of about 35 datapoints and no further significant decrease beyond that.

In the next set of computation experiments, the dataset size is fixed at 35 datapoints and the minimum zone error is evaluated versus the search space size $E$ : a square of side 2 $E$ from 1 to $0.02 \mathrm{~mm}$.

From Fig.5, the average error obtained from 30 runs decreases from 0.06082 to $0.06002 \mathrm{~mm}$, and particularly the standard deviation suffers a sharp decrease from 0.00256 to $0.00001 \mathrm{~mm}$, so that errors in the various repetitions are better gathered around the average error.

This result is not surprising, because in this computation experiment the search space is centered about the minimum zone center $(0,0)$, which is known; however this is not the case in general. In center-based minimum zone algorithms, a first raw estimation of the circular profile center is necessary

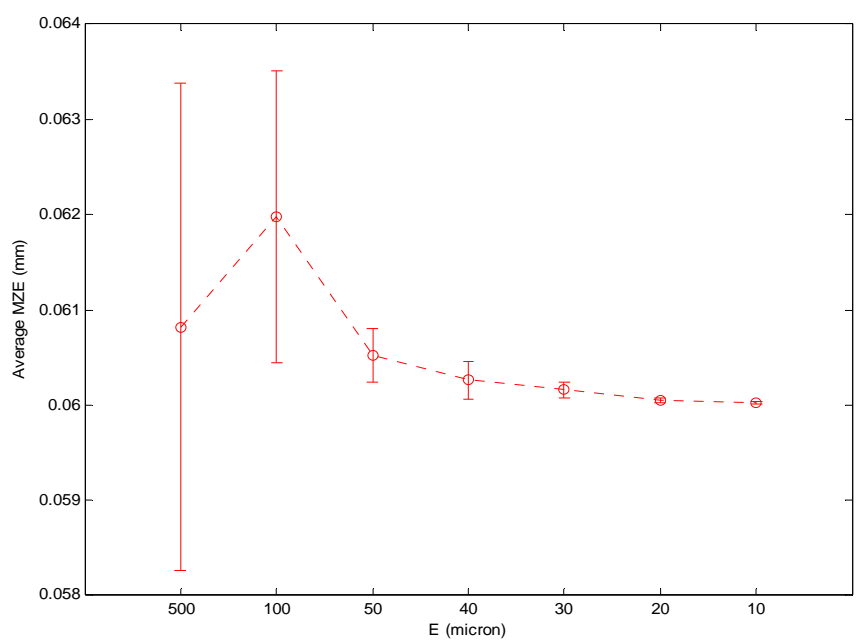

Fig.5. Average MZ error with error bars from 30 repetitions versus search space size, with dataset size 35 datapoints, for roundness error of $0.06 \mathrm{~mm}$. 
as the starting point for a more accurate roundness evaluation; usually the centroid or the least squares center are considered. In Fig. 6 the centroid position is shown for different dataset sizes and it can be noticed that its distance from the MZ can be as high as $4 \mathrm{~mm}$ and over, yielding an $\mathrm{MZ}$ error double than that.

In the final set of experiments the $M Z E$ is evaluated by the genetic algorithm as a function of the dataset size (from 10 to 500 datapoints) and of the search space (from 0.1 to 10 $\mathrm{mm}$ or from 0.5 to $50 \%$ of the nominal radius $R$ ). 3D plots are available from Fig.7 to Fig.9 (and detailed results are reported from Table 2 to Table 4 of Appendix).

In Fig. 7 and in Fig.8 an area with minimum $M Z E$ and minimum standard deviation respectively are present. Other homogeneous regions can be identified and are discussed in the next chapter.

The computation time in Fig.9 increases approximately with the search space size and for a given search space it increases linearly with the dataset size as previously shown.

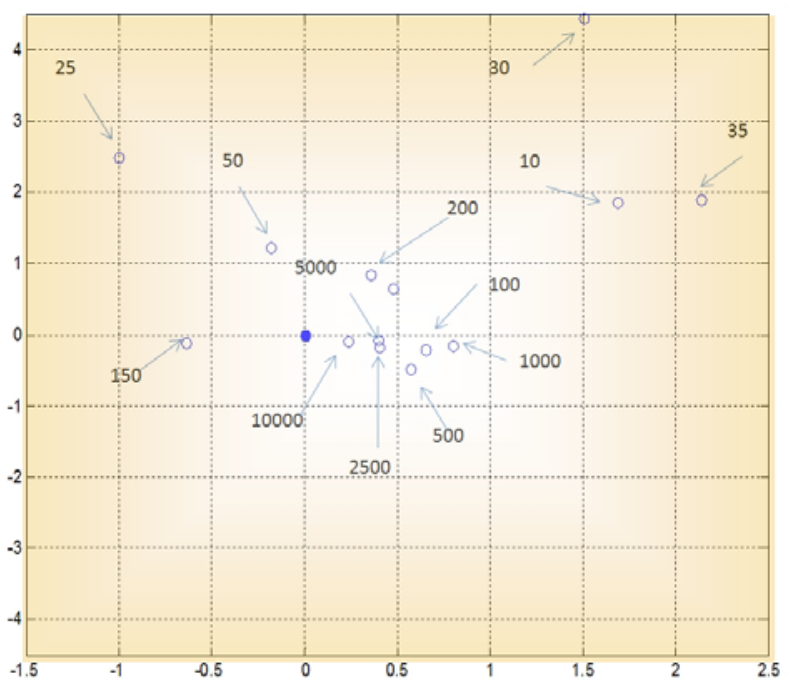

Fig.6. Centroids $(x, y)$ in $[\mathrm{mm}]$ for different dataset with size from 10 to 10,000 datapoints.

\section{RESULTS}

Small datasets (10 to 35 points) and small search spaces $(0.1$ to $1 \mathrm{~mm})$ produce high $M Z E$ on average. In fact, the centroid is far enough from the MZ center and the search space is too small to include it. With small datasets and larger search spaces (2 to $4 \mathrm{~mm}$ ), the average $\mathrm{MZ}$ error decreases as the search space gets larger, in a first range, bringing to better results, but then it increases because of higher probability of being trapped in local minima. With larger datasets (50 to 500 points) better results are achieved for small search spaces and the $\mathrm{MZ}$ error decreases as the search space increases up to $2 \mathrm{~mm}$. However, results get worse for larger search spaces. Larger datasets yield a more precise estimation of the center but too large search spaces are dangerous because it is easier to be trapped in local minima. It is speculated that the population size (pop) should be increased accordingly.

Good performance are achieved with medium sized search spaces (1 to $2 \mathrm{~mm}$ ) corresponding to datasets from 100 to 500 points: the average MZ error is about 0.062 -

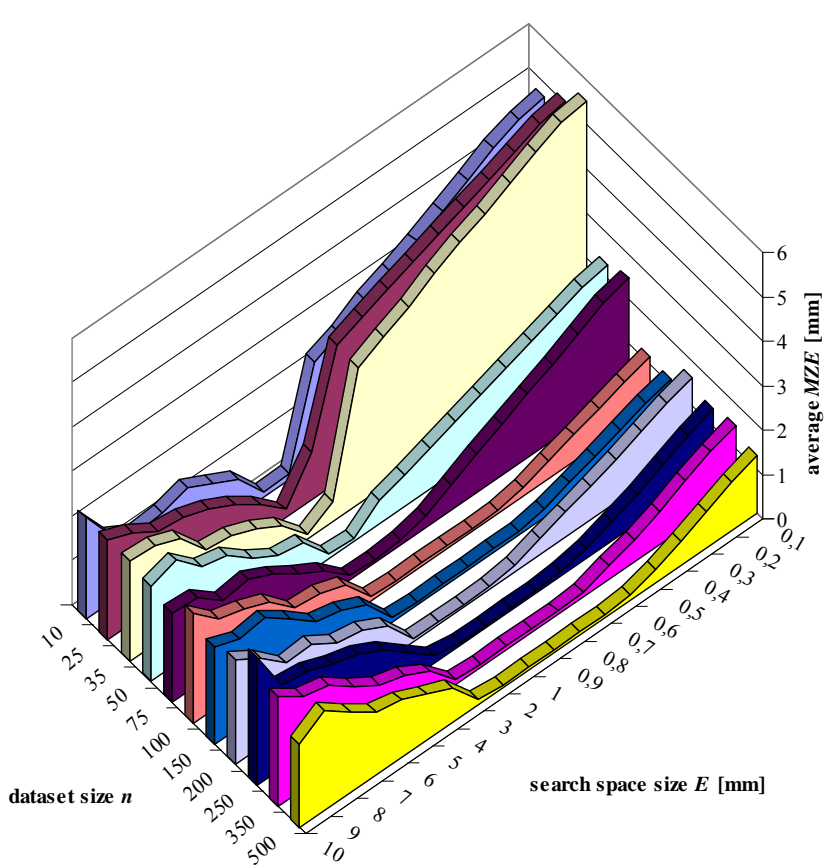

Fig.7. MZ error versus dataset size (10 to 500 datapoints) and search space size (0.1 to $10[\mathrm{~mm}])$. Each experimental datum is the result of 30 runs and is detailed in Table 2 of Appendix.

$0.063 \mathrm{~mm}$, a pretty good approximation of the real roundness error of $0.06 \mathrm{~mm}$, and the standard deviation is about 0.036 to $0.04 \mathrm{~mm}$. With larger datasets (datapoints > 500 ) with search space centered in the centroid of size 1 to 2 $\mathrm{mm}$, the average $\mathrm{MZ}$ error is similar to that achieved in the preliminary analysis considering the ideal search space centered in the known MZ center with size $0.5 \mathrm{~mm}: 0.06310$ $\mathrm{mm}$ (1,000 points), $0.06262 \mathrm{~mm}$ (2,500 points), 0.06332 $\mathrm{mm}$ (5,000 points), $0.06170 \mathrm{~mm}$ (10,000 points).

The optimum dataset size and search space are

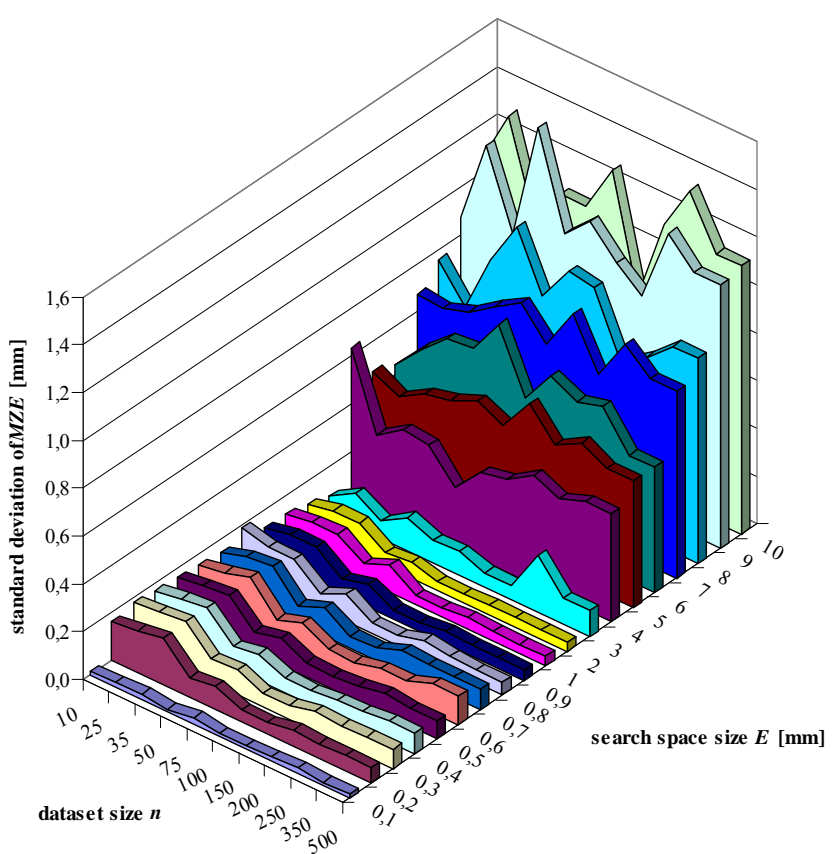

Fig.8. Standard deviation of the MZ error versus dataset size (10 to 500 datapoints) and search space size $(0.1$ to $10[\mathrm{~mm}])$. Each experimental datum is the result of 30 runs and is detailed in Table 3 of Appendix. 


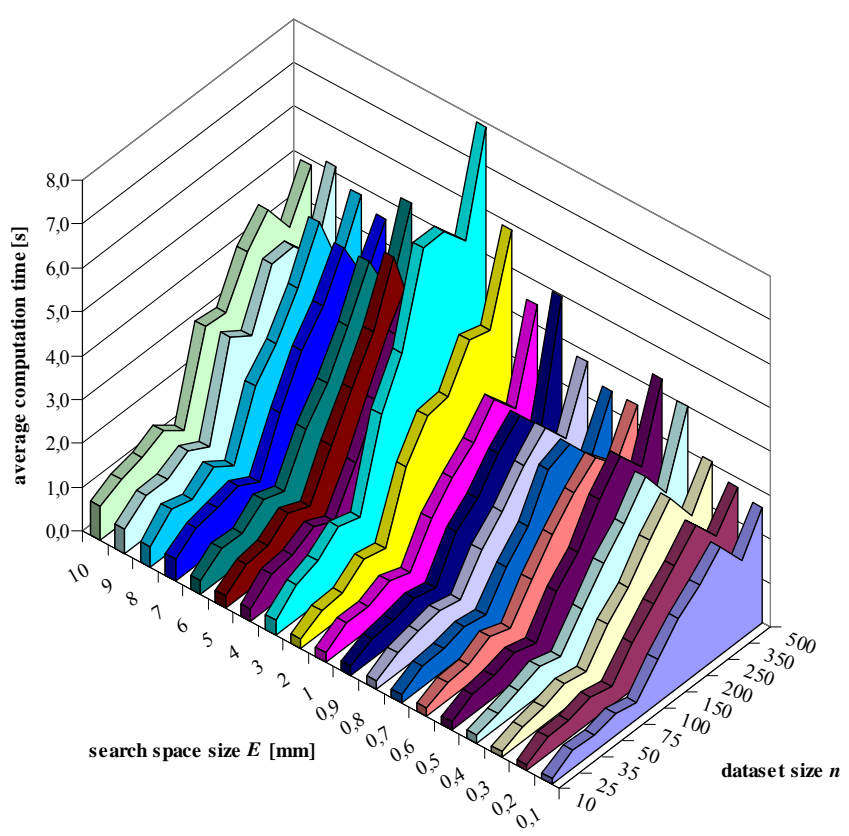

Fig.9. Computation time versus dataset size (10 to 500 datapoints) and search space size $(0.1$ to $10[\mathrm{~mm}])$. Each experimental datum is the result of 30 runs and is detailed in Table 4 of Appendix.

respectively 100 and $2 \mathrm{~mm}$, and the average $\mathrm{MZ}$ error obtained is $0.06209 \mathrm{~mm}$ with standard deviation of 0.03564 $\mathrm{mm}$.

From Fig.8, the standard deviation is generally small for small search spaces, and it increases with the search space size.

\section{DISCUSSION}

The initial intent of current work was to focus on very large datasets, which can be obtained by scanning techniques or optical methods. It was expected that by a higher amount of data the MZ estimation would be more accurate, which is not confirmed experimentally on artificially generated datasets. The MZ error decreases with the dataset size only up to a given value. Unexpectedly with large dataset sizes (from 500 to 10,000 acquired points) the MZ error does not improve significantly, with a linear increase in computation time, independently on the $\mathrm{MZ}$ error. Whereas, with small datasets (from 75 to 500 points) improvements are clearly visible, both in term of mean and standard deviation of MZ errors from 30 trials in each experimental condition tested.

The search space size has been defined as a square centered in the centroid of the sampled profile. Error maps as a function of dataset size and search space show a typical pattern, with a minimum.

The high MZ error can be explained in a different way for small and large search spaces. With small search spaces, the algorithm stops after few runs because the MZ center is not included and the algorithm is easily trapped in local minima. Whereas with high search space solutions are sparse and the algorithm requires more iterations until it is trapped again in a local minimum.

Taking into account the processing time by comparing Fig.7 and Fig.9, it can be noticed that with a 75 points dataset and a search space of $2 \mathrm{~mm}$ the average $\mathrm{MZ}$ error
$(0.06398 \mathrm{~mm})$ is close to the value for a 100 points dataset and the same search space $(0.06209 \mathrm{~mm}$, the minimum value achieved), but with the 75 points dataset the computation time is reduced from 2.2973 to $0.83605 \mathrm{~s}$.

Being sensitive to small geometrical parameters changes, the computation time should also be put into consideration in the two parameters selection for overall performance improvement.

More generally, this pattern is helpful to take out indications on the right number of points and search space conjugation in a roundness evaluation problem.

The industrial relapse from this analysis is to upper limit the number of acquired datapoints for the tolerance estimation in the process setup phase: unnecessary datapoints may have a strong impact on processing time, without accuracy improvement.

\section{CONCLUSIONS}

In this work, a genetic algorithm for the roundness evaluation of circular profiles using the MZT method has been optimized. In particular, two geometric parameters have been considered: the dataset and the search space sizes. These parameters are fundamental to make the algorithm properly work in an automated production environment, because of their strong influence on performance in terms of estimation error and computation time ( 0.1 to more than 2 seconds with the experimental datasets tested). A previously optimized genetic algorithm with certified datasets has been used in computation experiments.

From a cross analysis, by varying both the geometric parameters, indications for the choice of optimal values are provided. Eventually, considerations about the computation times lead to a better choice of the geometric parameters that improves the overall algorithm performance.

The extensive experimental results and the optimization approach presented provide a strong base for both future theoretical investigations and for direct industrial application of the proposed GA parameters as well.

\section{APPENDIX}

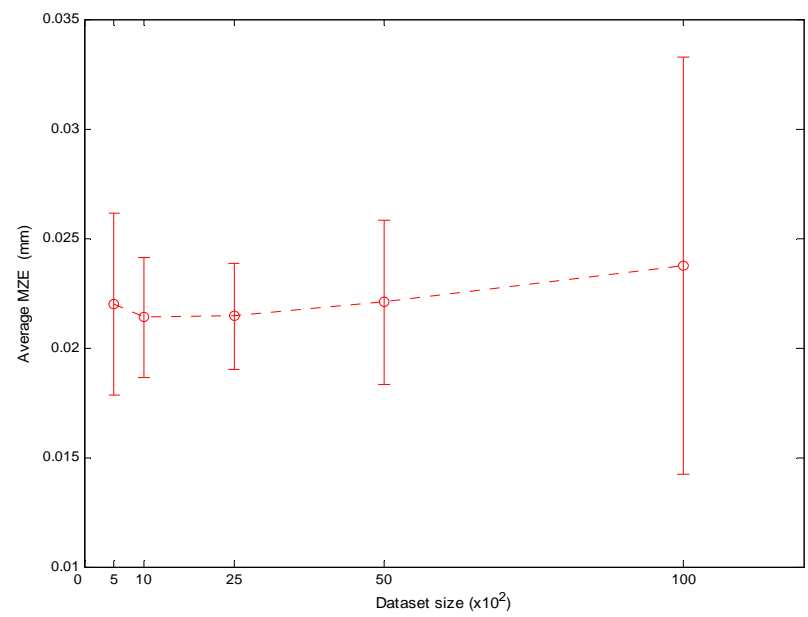

Fig.10. Average $M Z E$ with error bars from 30 repetitions versus dataset size, for roundness error of $0.02 \mathrm{~mm}$. 


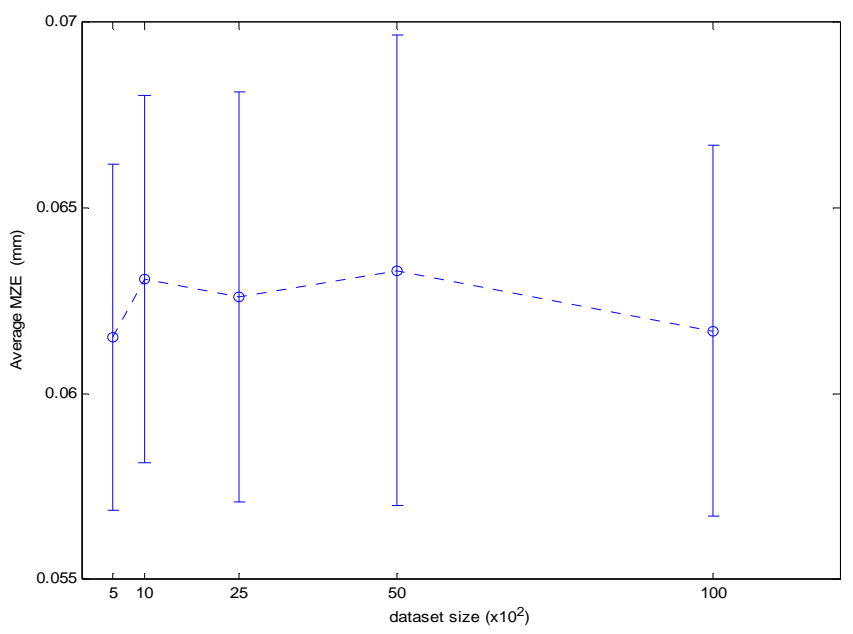

Fig.11. Average $M Z E$ with error bars from 30 repetitions versus dataset size, for roundness error of $0.06 \mathrm{~mm}$.

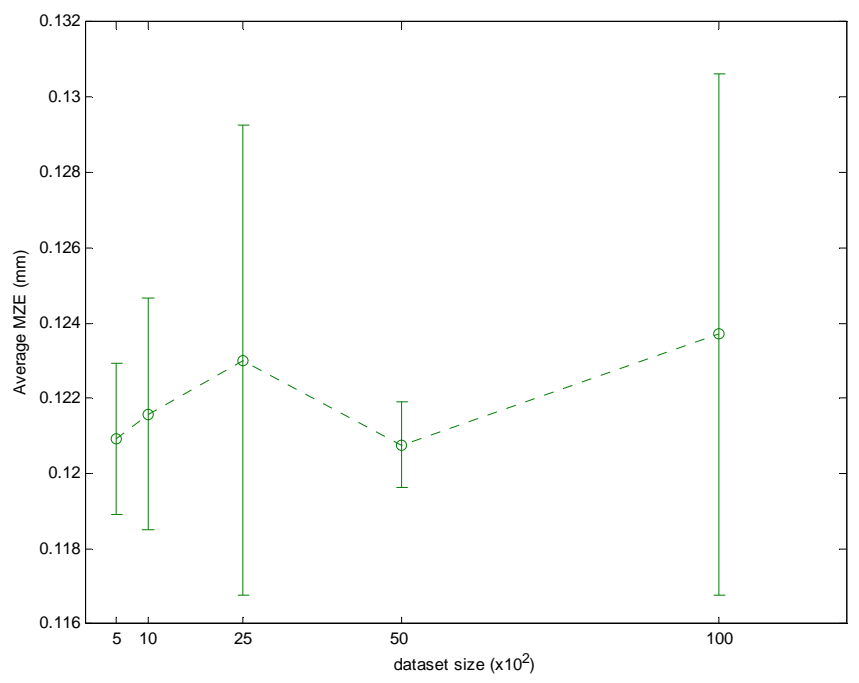

Fig.12. Average $M Z E$ with error bars from 30 repetitions versus dataset size, for roundness error of $0.12 \mathrm{~mm}$.

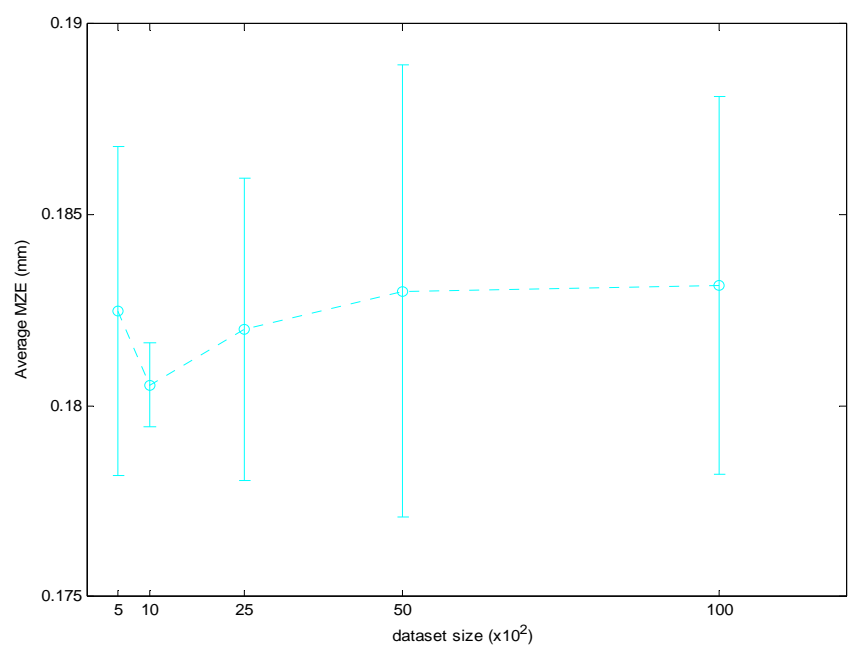

Fig.13. Average $M Z E$ with error bars from 30 repetitions versus dataset size, for roundness error of $0.18 \mathrm{~mm}$.
Table 2. Average MZ error versus dataset size (10 to 500 datapoints) and search space size $(0.1$ to $10[\mathrm{~mm}])$. Each experimental datum is the result of 30 runs. Color codes for MZ errors [mm]: $0.060-0.063,0.064-0.07,0.08-0.10,0.11-0.50$.

\begin{tabular}{|c|rrrrrrrrrrr|}
\cline { 2 - 11 } \multicolumn{1}{c|}{} & 10 & 25 & 35 & 50 & 75 & 100 & 150 & 200 & 250 & 350 & 500 \\
\hline 0,1 & 4,67 & 5,04 & 5,51 & 2,31 & 2,47 & 1,19 & 1,14 & 1,62 & 1,40 & 1,49 & 1,31 \\
0,2 & 4,57 & 4,93 & 5,43 & 2,18 & 2,29 & 1,00 & 0,98 & 1,45 & 1,19 & 1,30 & 1,09 \\
0,3 & 4,31 & 4,68 & 5,16 & 1,98 & 2,00 & 0,79 & 0,79 & 1,21 & 0,93 & 1,04 & 0,82 \\
0,4 & 3,97 & 4,46 & 4,86 & 1,76 & 1,71 & 0,59 & 0,57 & 0,96 & 0,65 & 0,74 & 0,54 \\
0,5 & 3,69 & 4,25 & 4,55 & 1,56 & 1,44 & 0,39 & 0,37 & 0,75 & 0,43 & 0,48 & 0,30 \\
0,6 & 3,39 & 4,02 & 4,32 & 1,34 & 1,15 & 0,19 & 0,18 & 0,57 & 0,22 & 0,32 & 0,12 \\
0,7 & 3,11 & 3,80 & 3,99 & 1,14 & 0,88 & 0,08 & 0,08 & 0,36 & 0,10 & 0,11 & 0,08 \\
0,8 & 2,86 & 3,58 & 3,64 & 0,93 & 0,56 & 0,065 & 0,064 & 0,16 & 0,07 & 0,068 & 0,065 \\
0,9 & 2,50 & 3,33 & 3,36 & 0,73 & 0,27 & 0,063 & 0,063 & 0,069 & 0,064 & 0,063 & 0,064 \\
1 & 2,24 & 3,09 & 3,07 & 0,53 & 0,12 & 0,063 & 0,063 & 0,068 & 0,063 & 0,064 & 0,063 \\
2 & 0,14 & 1,08 & 0,42 & 0,08 & 0,064 & 0,062 & 0,062 & 0,062 & 0,062 & 0,062 & 0,062 \\
3 & 0,10 & 0,18 & 0,08 & 0,12 & 0,09 & 0,09 & 0,09 & 0,08 & 0,19 & 0,10 & 0,12 \\
4 & 0,79 & 0,68 & 0,67 & 0,78 & 0,60 & 0,76 & 0,78 & 0,78 & 0,64 & 0,67 & 0,78 \\
5 & 1,08 & 1,08 & 1,02 & 0,86 & 0,92 & 0,96 & 1,05 & 1,05 & 1,08 & 0,98 & 1,02 \\
6 & 1,39 & 1,38 & 1,25 & 1,20 & 1,31 & 1,01 & 1,17 & 1,18 & 1,40 & 1,15 & 1,29 \\
7 & 1,21 & 1,57 & 1,31 & 1,67 & 1,58 & 1,56 & 1,63 & 1,52 & 1,56 & 1,52 & 1,43 \\
8 & 1,15 & 1,66 & 1,97 & 1,81 & 1,50 & 1,78 & 2,04 & 1,50 & 1,79 & 1,95 & 1,82 \\
9 & 1,48 & 2,02 & 2,21 & 2,33 & 1,95 & 1,94 & 1,96 & 1,99 & 1,87 & 2,07 & 2,23 \\
10 & 2,27 & 2,26 & 2,23 & 2,15 & 2,01 & 2,49 & 2,18 & 2,35 & 2,90 & 2,46 & 1,85 \\
\hline
\end{tabular}

Table 3. Standard deviation of the MZ error versus dataset size (10 to 500 datapoints) and search space size $(0.1$ to $10[\mathrm{~mm}])$. Each experimental datum is the result of 30 runs. Color codes for error [mm]: 0.010-0.023, 0.024-0.040, 0.041-0.050, 0.051-1.000, 1.0011.500 .

\begin{tabular}{|r|rrrrrrrrrrr|}
\multicolumn{1}{l|}{} & 10 & 25 & 35 & 50 & 75 & 100 & 150 & 200 & 250 & 350 & 500 \\
\hline 0,1 & 0,017 & 0,020 & 0,023 & 0,010 & 0,028 & 0,014 & 0,011 & 0,015 & 0,023 & 0,014 & 0,014 \\
0,2 & 0,162 & 0,175 & 0,193 & 0,082 & 0,095 & 0,049 & 0,048 & 0,069 & 0,062 & 0,064 & 0,064 \\
0,3 & 0,180 & 0,193 & 0,205 & 0,097 & 0,102 & 0,057 & 0,060 & 0,084 & 0,076 & 0,080 & 0,080 \\
0,4 & 0,169 & 0,186 & 0,201 & 0,090 & 0,109 & 0,063 & 0,060 & 0,072 & 0,077 & 0,086 & 0,086 \\
0,5 & 0,161 & 0,179 & 0,190 & 0,089 & 0,116 & 0,065 & 0,058 & 0,069 & 0,088 & 0,077 & 0,077 \\
0,6 & 0,153 & 0,173 & 0,204 & 0,079 & 0,115 & 0,060 & 0,055 & 0,082 & 0,080 & 0,119 & 0,119 \\
0,7 & 0,144 & 0,176 & 0,187 & 0,075 & 0,119 & 0,052 & 0,054 & 0,096 & 0,086 & 0,086 & 0,086 \\
0,8 & 0,188 & 0,158 & 0,158 & 0,066 & 0,115 & 0,043 & 0,043 & 0,076 & 0,061 & 0,056 & 0,056 \\
0,9 & 0,126 & 0,146 & 0,147 & 0,064 & 0,094 & 0,039 & 0,039 & 0,052 & 0,046 & 0,044 & 0,044 \\
1 & 0,119 & 0,136 & 0,141 & 0,067 & 0,114 & 0,037 & 0,037 & 0,048 & 0,040 & 0,039 & 0,039 \\
2 & 0,099 & 0,125 & 0,125 & 0,051 & 0,063 & 0,036 & 0,036 & 0,041 & 0,037 & 0,037 & 0,037 \\
3 & 0,074 & 0,137 & 0,072 & 0,139 & 0,094 & 0,099 & 0,061 & 0,061 & 0,237 & 0,110 & 0,110 \\
4 & 0,634 & 0,322 & 0,394 & 0,380 & 0,251 & 0,370 & 0,391 & 0,458 & 0,411 & 0,453 & 0,453 \\
5 & 0,473 & 0,419 & 0,480 & 0,507 & 0,554 & 0,506 & 0,647 & 0,526 & 0,585 & 0,529 & 0,529 \\
6 & 0,437 & 0,556 & 0,651 & 0,661 & 0,815 & 0,557 & 0,700 & 0,645 & 0,676 & 0,524 & 0,524 \\
7 & 0,675 & 0,664 & 0,703 & 0,776 & 0,844 & 0,724 & 0,898 & 0,698 & 0,874 & 0,781 & 0,781 \\
8 & 0,757 & 0,610 & 0,856 & 1,045 & 0,811 & 0,966 & 0,947 & 0,686 & 0,759 & 0,857 & 0,857 \\
9 & 0,873 & 1,222 & 0,905 & 1,404 & 1,007 & 1,111 & 0,992 & 0,905 & 1,217 & 1,102 & 1,102 \\
10 & 1,053 & 1,286 & 0,916 & 1,065 & 1,064 & 1,266 & 0,790 & 1,144 & 1,336 & 1,121 & 1,121 \\
\hline
\end{tabular}

Table 4. Computation time [s] versus dataset size (10 to 500 datapoints) and search space size $(0.1$ to $10[\mathrm{~mm}])$. Each experimental datum is the result of 30 runs. Color codes for times [s]: $0.13-0.50,0.51-1.00,1.01-1.50,1.51-2.00,2.01-2.50,2.51-$ 3.50 .

\begin{tabular}{|c|llllllllllll|}
\hline \multicolumn{1}{c|}{} & 10 & 25 & 35 & 50 & 75 & 100 & 150 & 200 & 250 & 350 & 500 \\
\hline 0,1 & 0.14 & 0.41 & 0.39 & 0.51 & 0.39 & 1.02 & 1.56 & 1.99 & 2.48 & 1.81 & 2.61 \\
0,2 & 0.14 & 0.40 & 0.41 & 0.58 & 0.45 & 1.07 & 1.60 & 2.11 & 2.65 & 2.03 & 2.79 \\
0,3 & 0.14 & 0.33 & 0.41 & 0.59 & 0.48 & 1.18 & 1.68 & 2.37 & 2.97 & 2.18 & 3.05 \\
0,4 & 0.20 & 0.34 & 0.47 & 0.66 & 0.52 & 1.24 & 1.90 & 2.45 & 3.16 & 2.41 & 4.02 \\
0,5 & 0.19 & 0.45 & 0.50 & 0.68 & 0.52 & 1.32 & 1.85 & 2.69 & 3.31 & 2.69 & 4.31 \\
0,6 & 0.20 & 0.41 & 0.45 & 0.72 & 0.55 & 1.30 & 1.95 & 2.52 & 2.97 & 2.44 & 3.41 \\
0,7 & 0.19 & 0.48 & 0.51 & 0.65 & 0.53 & 1.27 & 1.80 & 2.73 & 2.98 & 2.32 & 3.42 \\
0,8 & 0.20 & 0.49 & 0.60 & 0.71 & 0.58 & 1.34 & 1.85 & 2.54 & 3.02 & 2.41 & 3.81 \\
0,9 & 0.23 & 0.48 & 0.55 & 0.65 & 0.55 & 1.44 & 1.85 & 2.48 & 3.01 & 2.39 & 4.97 \\
1 & 0.23 & 0.54 & 0.53 & 0.75 & 0.58 & 1.43 & 1.98 & 2.42 & 3.05 & 2.47 & 4.48 \\
2 & 0.23 & 0.50 & 0.54 & 0.94 & 0.84 & 2.30 & 3.10 & 3.16 & 4.09 & 3.93 & 5.86 \\
3 & 0.35 & 0.79 & 0.97 & 1.37 & 1.22 & 3.21 & 4.22 & 6.25 & 6.26 & 5.67 & 7.90 \\
4 & 0.29 & 0.72 & 0.79 & 1.06 & 0.83 & 1.79 & 2.85 & 3.94 & 4.75 & 3.97 & 4.99 \\
5 & 0.30 & 0.63 & 0.70 & 1.07 & 0.85 & 1.89 & 2.80 & 3.71 & 5.07 & 3.28 & 4.32 \\
6 & 0.32 & 0.70 & 0.65 & 1.05 & 0.89 & 1.92 & 2.62 & 3.55 & 4.66 & 3.55 & 5.24 \\
7 & 0.51 & 0.84 & 0.93 & 1.07 & 0.78 & 1.99 & 3.05 & 3.69 & 4.66 & 3.57 & 4.56 \\
8 & 0.48 & 0.96 & 0.82 & 1.21 & 0.87 & 2.30 & 2.90 & 3.79 & 4.94 & 3.58 & 4.81 \\
9 & 0.56 & 0.97 & 0.99 & 1.27 & 0.98 & 3.09 & 2.76 & 4.03 & 4.05 & 3.48 & 5.16 \\
10 & 0.80 & 1.03 & 1.17 & 1.42 & 1.05 & 3.06 & 2.99 & 3.99 & 4.58 & 3.83 & 4.88 \\
\hline
\end{tabular}




\section{ACKNOWLEDGMENT}

Work developed as Meo and Profumo's project work in Manufacturing Automation of the 2010-11 MEng degree in Automation Engineering at Pisa University.

\section{REFERENCES}

[1] International Organization for Standardization (2011). ISO 1101, Geometrical Product Specifications (GPS)-tolerances of form, orientation, location and run out, 2nd ed., Geneva, Switzerland.

[2] ASME B89.3.1 (2003) Measurement of Out-OfRoundness.

[3] Sharma, R., Rajagopal, K. \& Anand, S. (2000). A genetic algorithm based approach for robust evaluation of form tolerances, Journal of Manufacturing Systems, 19(1), 46-57.

[4] Dhanish, P.B. \& Shunmugam, M.S. (1991). An algorithm for form error evaluation - using the theory of discrete and linear chebyshev approximation, Computer Methods in Applied Mechanics and Engineering, 92, 309-324.

[5] Murthy, T.S.R. \& Abdin, S.Z. (1980). Minimum zone evaluation of surfaces, Int. J. of Machine Tool Design Research, 20, 123-136.

[6] Kovvur, Y., Ramaswami, H., Anand R.B. \& Sam Anand (2008). Minimum-zone form tolerance evaluation using particle swarm optimisation, Int. $J$. Intelligent Systems Technologies and Applications, 4(1/2).

[7] Weber, T., Motavalli, S., Fallahi, B., \& Cheraghi, S.H. (2002). A unified approach to form error evaluation, Journal of the International Societies for Precision Engineering and Nanotechnology, 26, 269-278.

[8] Wen, X., Xia, Q. \& Zhao, Y. (2006). An effective genetic algorithm for circularity error unified evaluation, Int. J. of Machine Tools \& Manufacture, 46, 1770-1777.

[9] Yan, L., Yan, B., Cai, L., Hu, G. \& Wang, M. (2009). Research on roundness error evaluation of shaft parts based on genetic algorithms with transfer-operator, ICEMI 2009 - Proceedings of Nineth International Conference on Electronic Measurement and Instruments, 2362-2366.

[10] Rossi, A., Antonetti, M., Barloscio M. \& Lanzetta, M. (2011). Fast genetic algorithm for roundness evaluation by the minimum zone tolerance (MZT) method, Measurement, 44(7), 1243-1252.

[11] Rossi, A. \& Lanzetta, M. (2013). Optimal blind sampling strategy for minimum zone roundness evaluation by metaheuristics, Precision Engineering, 37, 241-247, doi:10.1016/j.precisioneng.2012.09.001.

[12] Moroni, G. \& Petrò, S. (2008). Geometric tolerance evaluation: A discussion on minimum zone fitting algorithms, Precision Engineering, 32(3), 232-237.

[13] Chajda, J., Grzelka, M., Gapinski, B., Pawłowski, M. Szelewski, M. \& Rucki, M. (2008). Coordinate measurement of complicated parameters like roundness, cylindricity, gear teeth or free-form surface, $8^{\text {th }}$ Int. Conf. on Advanced Manufacturing Operations.

[14] Gapinski, B, \& Rucki, M. (2007). Uncertainty in CMM Measurement of Roundness, AMUEM 2007 International Workshop on Advanced Methods for Uncertainty Estimation in Measurement Sardegna, Trento, Italy, 16-18 July 2007.

[15] Gapinski, B, Grezelka, M. \& Rucki, M. (2006). Some aspects of the roundness measurement with CMM, XVIII IMEKO World Congress Metrology for a Sustainable Development September, 17 - 22, 2006, Rio de Janeiro, Brazil.

[16] Rossi, A. (2001). A form of deviation-based method for coordinate measuring machine sampling optimization in an assessment of roundness, Proc. Instn Mech. Engrs, Part B: Journal of Engineering Manufacture, 215, 1505-1518.

[17] Rossi, A. (2001). A minimal inspection sampling technique for roundness evaluation, 1st CIRP International Seminar on PRogress in Innovative Manufacturing Engineering (Prime), Sestri Levante, Italy, June, 20-22, 2001, 189-196.

[18] Weckenmann, A., Knauer, M., \& Kunzmann, H. (1998). The influence of measurement strategy on the uncertainty of CMM-measurements. CIRP AnnalsManufacturing Technology, 47(1), 451-454.

[19] Weckenmann, A., Weber, H., Eitzert, H. \& Garmer, M. (1995). Functionality-oriented evaluation and sampling strategy in coordinate metrology, Precision Engineering, 17(4), 244-52.

[20] Rossi, A. \& Lanzetta, M. (2013). Roundness: a closed form upper bound for the centroid to minimum zone center distance by worst-case analysis, Measurement, 46 (7), 2251-2258, doi:10.1016/j.measurement.2013.03.025.

[21] National Physical Laboratory (UK), Data Generator for Chebyshev Best-Fit Circle, http://www.npl.co.uk/ mathematics-scientific-computing/software-supportfor-metrology/, acc. 05/2013. 